\title{
Comprehensive Analysis of the Microbiome of the Horny Plug, and Interaction of Malassezia Species and Propionibacterium acnes in Keratinocytes
}

P329

○Otomi Cho ${ }^{1}$, Unno Mizuki ${ }^{1}$, Takashi Sugita ${ }^{1}$

1.Department of Microbiology, Meiji Pharmaceutical University

\section{Introduction}

Both Malassezia and Propionibacterium acnes are found in the horny plug and the overgrowth of these microorganisms can result in inflammation. A better understanding of the microbiome of the horny plug would thus provide insights into the pathology of Malassezia and $P$. acnes. Although $P$. acnes is known to induce the production of proinflammatory cytokines by keratinocytes in vitro, the mechanisms underlying the regulation of $P$. acnesinduced inflammatory responses are unclear.

In this study, the microbiome of the horny plug was analyzed comprehensively and the effect of Malassezia species and $P$. acnes on cytokine production by keratinocytes (NHEK cells) investigated.

\section{Materials and Methods}

\section{1) Sample collection \\ One hundred horny plug samples were collected from 10 healthy Japanese individuals (6 males and 4 females).}

2) Microbiota analysis

3) Quantitative analysis

The DNA of Malassezia species and $P$. acnes was quantified using genus specific primers and TaqMan probes.

4) Enzyme-Linked immunosorbent assay

Malassezia species and $P$. acnes were cultured in the presence or absence of a keratinocyte cell line (NHEK), and the production of interleukin (IL)-6, IL-1 $\alpha$, IL-8 and tumor necrosis factor (TNF)- $\alpha$ by the keratinocytes was examined.

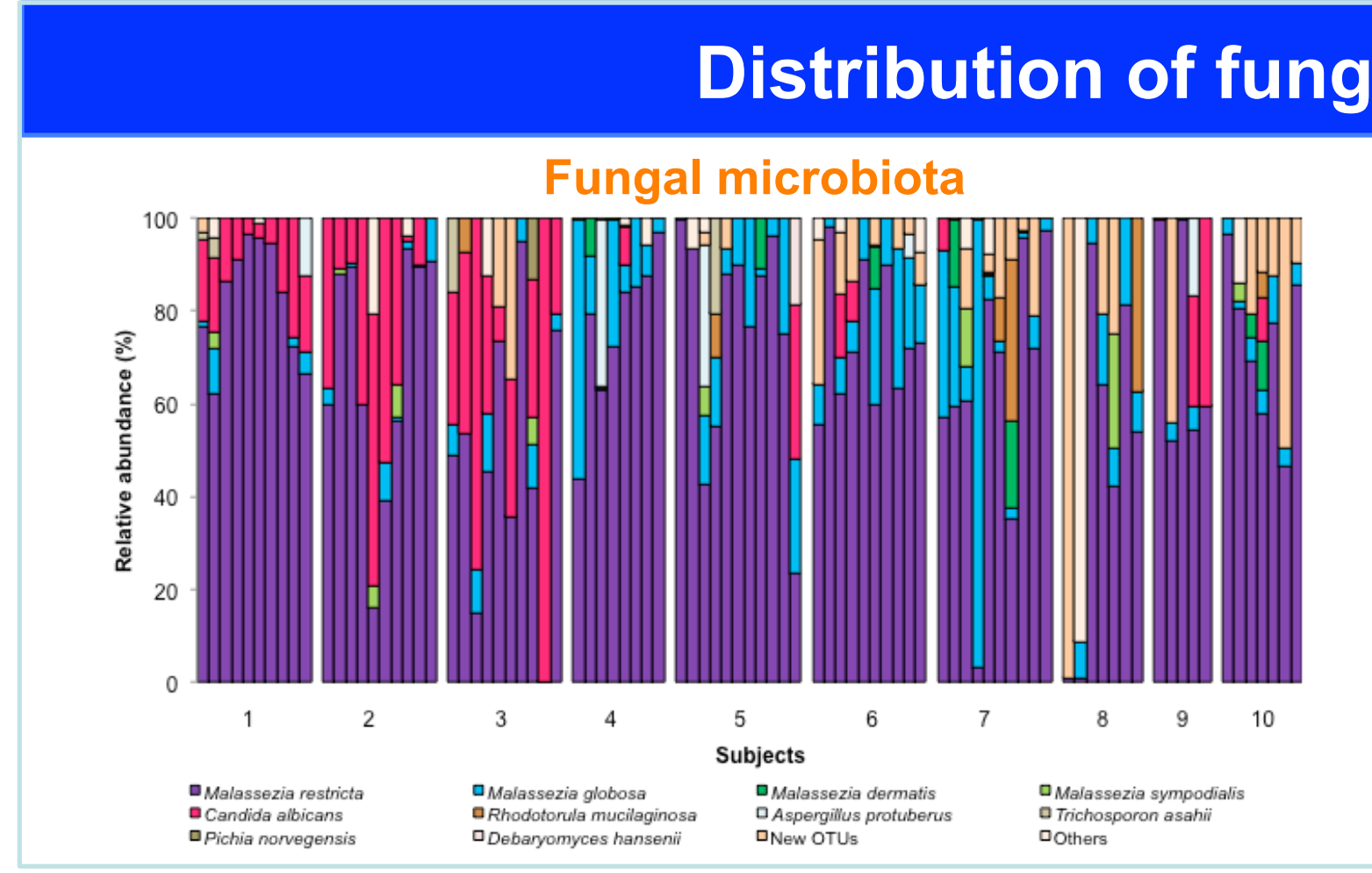

\section{Localization of fungal and bacteria in plug}
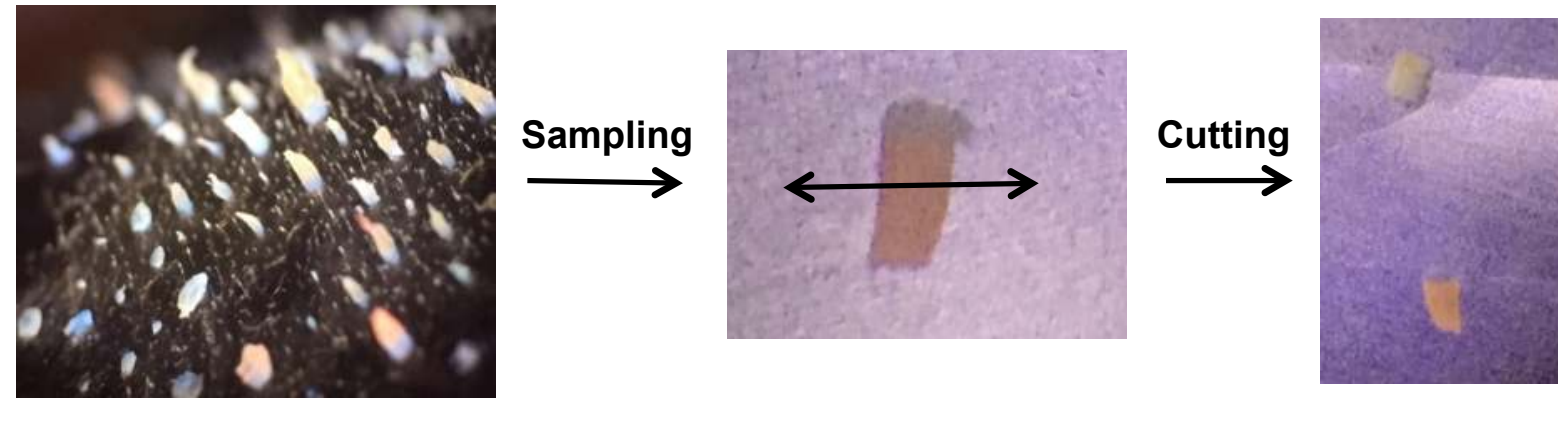

Outside

Outside

Inside
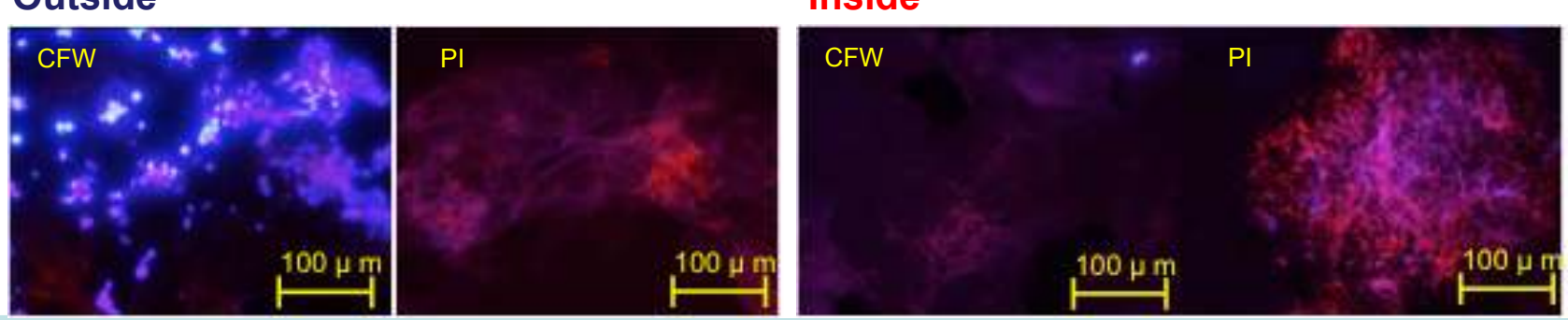

Malassezia increase $\boldsymbol{P}$. acnes-induced inflammatory Response of keratinocyte
IL-6
$\mathrm{IL}-1 \alpha$
IL-8

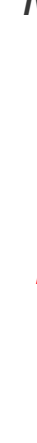

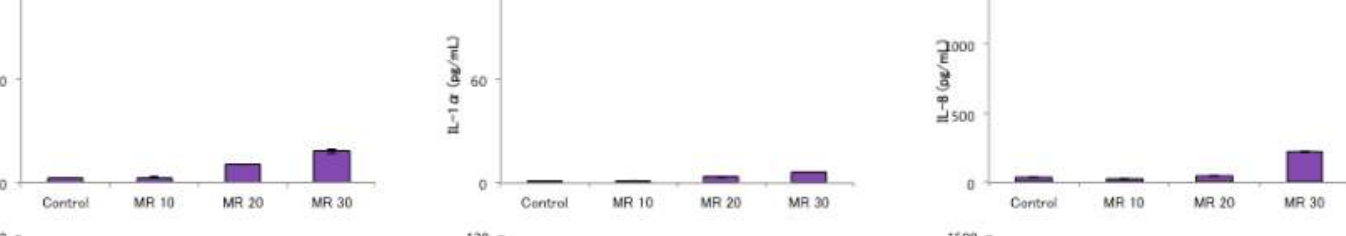$$
\text { . acnes }
$$

Co-culture
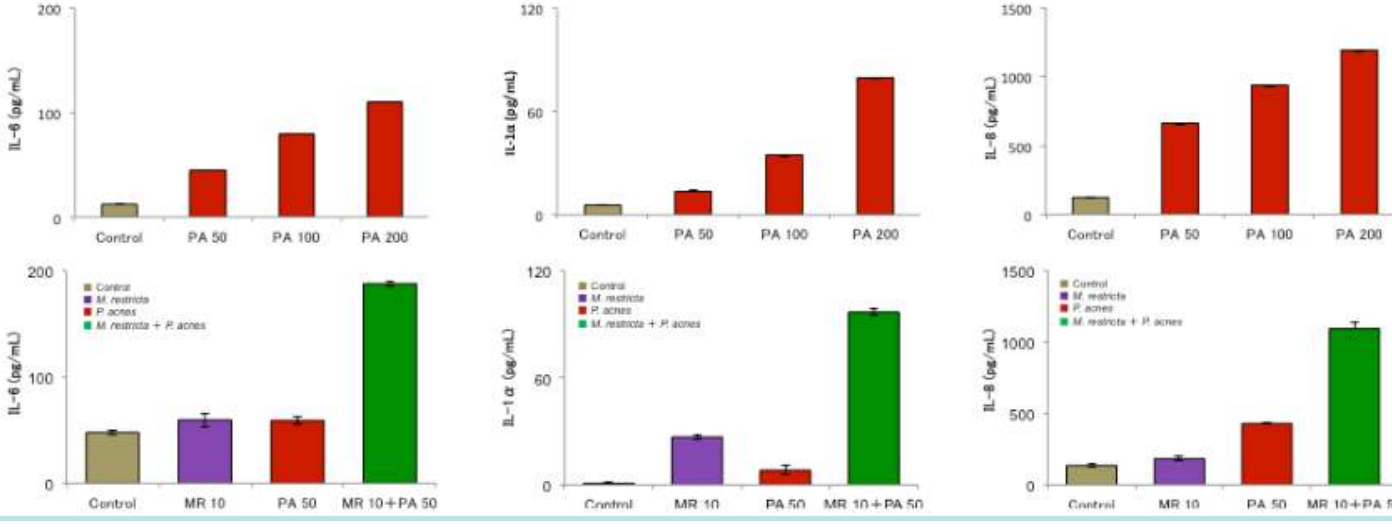

\section{Quantitative real-time PCR analysis}

Bacterial microbiota

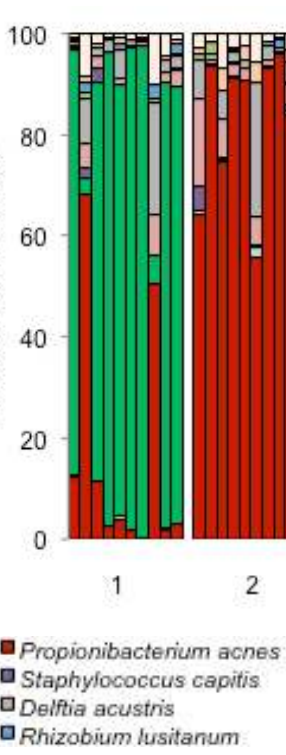



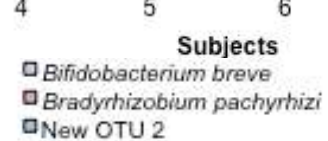

PCoA analysis

Fungal microbiota

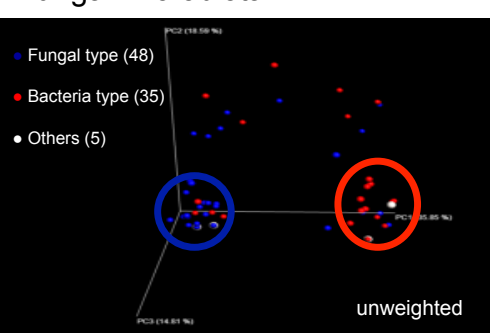

Bacteria microbiota

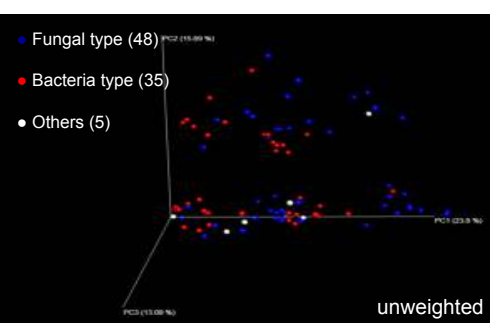

$-8$

